\title{
MODELLING AND SIMULATION OF HIGH-SPEED MACHINING
}

\author{
T. D. MARUSICH AND M. ORTIZ \\ Division of Engineering, Brown University, Providence RI 02912, U.S.A.
}

\begin{abstract}
SUMMARY
A Lagrangian finite element model of orthogonal high-speed machining is developed. Continuous remeshing and adaptive meshing are the principal tools which we employ for sidestepping the difficulties associated with deformation-induced element distortion, and for resolving fine-scale features in the solution. The model accounts for dynamic effects, heat conduction, mesh-on-mesh contact with friction, and full thermomechanical coupling. In addition, a fracture model has been implemented which allows for arbitrary crack initiation and propagation in the regime of shear localized chips. The model correctly exhibits the observed transition from continuous to segmented chips with increasing tool speed.
\end{abstract}

KEY WORDS: machining; finite elements; remeshing; thermo-mechanical coupling

\section{INTRODUCTION}

The development of a new machining process requires considerable investment of time and resources. ${ }^{1}$ Precise knowledge of the optimal range of the cutting parameters is essential for a timely startup. Process features such as tool geometry and cutting speed directly influence chip morphology, cutting forces, final product dimensionality and tool life. Computer simulation of the cutting process can potentially reduce the number of design iterations and result in a substantial cost savings. Considerable effort has therefore been devoted to the development of computational models of high-speed machining. Of primary concern is the determination of the steadystate temperature distribution in the workpiece, tool and chip (e.g. References 2-4). Attempts to model the process of chip formation have for the most part been based on a predetermined line of separation between the workpiece and chip (e.g. References 5, and 6). Nodes on this line are separated -and the line 'unzipped' - when the tool tip is sufficiently close, or when a certain level of plastic strain is attained. Evidently, this simple approach is not capable of predicting surface roughness and chip morphology. Sekhon and Chenot, ${ }^{7}$ by contrast, have used mesh adaptivity to allow for an arbitrary surface of separation. However, elastic strains are not accounted for, which precludes the computation of residual stresses in the workpiece. In addition, the model of Sekhon and Chenot $^{7}$ does not account for fracture, which severely limits the types of chip morphologies which can be predicted. It should also be noted that the majority of simulations conducted in the past idealize the tool as rigid. The tool stiffness, is known to directly influence surface roughness and tool chatter. ${ }^{8}$

In this paper, we present a model of high-speed machining overcomes some of these limitations. High-speed machining invariably involves extremely high deformation rates and unconstrained plastic flow. Lagrangian codes based on a fixed mesh have difficulties dealing with this class of 
problems owing to the large element distortions which, inevitably, are incurred. Considerable effort has been devoted to the development of elements which perform well when severely distorted $^{9-15}$ A number of alternative formulations have also been proposed for the analysis of unconstrained flows, including arbitrary Lagrangian-Eulerian (ALE) methods ${ }^{16-22}$ and pseudoconcentration methods. ${ }^{23-25}$

However, Lagrangian codes can be used to simulate large unconstrained plastic flows provided that the solid is continuously remeshed. This is the avenue which we have pursued here. Continuous remeshing and adaptive meshing are the principal tools which we employ for sidestepping the difficulties associated with deformation-induced element distortion, and for resolving fine-scale features in the solution. We also resort to mesh adaptivity as a means of following the propagation of cracks along arbitrary paths through the chip. Simulations of high-speed machining of a structural steel are presented in Section 10. Extensive experimental data are available for this type of material. ${ }^{26-29}$ The ability of the model to predict the transition between continuous and segmented chips with increasing tool speed is particularly noteworthy.

\section{EQUATIONS OF MOTION}

Consider a solid initially occupying a reference configuration $B_{0}$, and a process of incremental loading whereby the deformation mapping over $B_{0}$ changes from $\phi_{n}$, at time $t_{n}$, to $\phi_{n+1}=\phi_{n}+\mathbf{u}$, at time $t_{n+1}=t_{n}+\Delta t$. Dynamic equilibrium is enforced at time $t_{n+1}$ weakly by recourse to the virtual work principle

$$
\int_{B_{0}} \mathbf{P}_{n+1}: \nabla_{0} \boldsymbol{\eta} \mathrm{d} V_{0}-\int_{B_{0}}\left(\mathbf{f}_{n+1}-\rho_{0} \mathbf{a}_{n+1}\right) \cdot \boldsymbol{\eta} \mathrm{d} V_{0}-\int_{\partial B_{0+}} \mathbf{t}_{n+1} \cdot \boldsymbol{\eta} \mathrm{d} S_{0}=0
$$

where $\mathbf{P}_{n+1}$ denotes the first Piola-Kirchhoff stress field at time $t_{n+1}, \mathbf{f}_{n+1}, \mathbf{a}_{n+1}$ and $\mathbf{t}_{n+1}$ are the corresponding body forces, accelerations and boundary tractions, respectively, $\rho_{0}$ is the mass density on the reference configuration, $\boldsymbol{\eta}$ is an admissible virtual displacement field, and $\nabla_{0}$ denotes the material gradient. Assume for now that a rule has been determined to update the stress field of the general form

$$
\mathbf{P}_{n+1}=\hat{\mathbf{P}}\left(\mathbf{F}_{n+1} ; \text { state at } t_{n}, \Delta t\right)
$$

where the deformation gradients

$$
\mathbf{F}_{n+1}=\nabla_{0} \phi_{n+1}
$$

are assumed given. Inserting (2) and (3) into (1) one obtains

$$
\int_{B_{0}} \hat{\mathbf{P}}\left(\nabla_{0} \boldsymbol{\phi}_{n+1} ; \text { state at } t_{n}, \Delta t\right): \nabla_{0} \boldsymbol{\eta} \mathrm{d} V_{0}-\int_{B_{0}}\left(\mathbf{f}_{n+1}-\rho_{0} \mathbf{a}_{n+1}\right) \cdot \boldsymbol{\eta} \mathrm{d} V_{0}-\int_{\partial B_{0 \tau}} \mathbf{t}_{n+1} \cdot \boldsymbol{\eta} \mathrm{d} S_{0}=0
$$

which can be solved for the updated deformation mapping $\phi_{n+1}$. Upon discretization of (2) with finite elements the governing equations become

$$
\mathbf{M a}_{n+1}+\mathbf{R}_{n+1}^{\text {int }}=\mathbf{R}_{n+1}^{\text {ext }}
$$

where

$$
M_{i a k b}=\int_{B_{0}} \rho_{0} \delta_{i k} N_{a} N_{b} \mathrm{~d} V_{0}
$$


is the mass matrix

$$
R_{i a}^{\text {ext }}=\int_{B_{0}} f_{i} N_{a} \mathrm{~d} V_{0}+\int_{\partial B_{0\ulcorner}} t_{i} N_{a} \mathrm{~d} S_{0}
$$

is the external force array and

$$
R_{i a}^{\mathrm{int}}=\int_{B_{0}} P_{i J} N_{a, J} \mathrm{~d} V_{0}
$$

is the internal force array. In the above expressions, $N_{a}, a=1, \ldots$, numnp are the shape functions.

The second-order accurate central difference scheme is used to discretize (5) in time, ${ }^{30-32}$ with the result

$$
\begin{aligned}
& \mathbf{d}_{n+1}=\mathbf{d}_{n}+\Delta t \mathbf{v}_{n}+\frac{1}{2} \Delta t^{2} \mathbf{a}_{n} \\
& \mathbf{a}_{n+1}=\mathbf{M}^{-1}\left(\mathbf{R}_{n+1}^{\text {ext }}-\mathbf{R}_{n+1}^{\text {int }}\right) \\
& \mathbf{v}_{n+1}=\mathbf{v}_{n}+\frac{1}{2} \Delta t\left(\mathbf{a}_{n+1}+\mathbf{a}_{n}\right)
\end{aligned}
$$

where $\mathbf{d}, \mathbf{v}$ and a denote the displacement, velocity and acceleration arrays, respectively. Although the minimum time step used in explicit dynamics is bounded by stability, ${ }^{33}$ contact algorithms available for explicit dynamics are more robust and straightforward than their implicit counterparts. Explicit algorithms are therefore more attractive for problems such as machining which involve complicated contact situations. Explicit integration is particularly attractive in threedimensional calculations, where implicit schemes lead to system matrices which often exceed the available in-core storage capacity. Yet another advantage of explicit algorithms is that they are ideally suited for concurrent computing. ${ }^{34}$

Explicit schemes require the use of diagonal or lumped mass matrices. No tangent stiffness matrix is assembled and no momentum iterations performed. The bulk of the computational costs lies in the constitutive calculations at the integration points and in the contact search. For computational efficiency in slow processes, the rate of loading (e.g. cutting tool speed) can be increased, or the density of the material can be artificially increased, which raises the stability limit. ${ }^{35}$ These techniques can be employed when inertia effects are not significant.

\section{SUBCYCLING}

Mesh refinement of the type described subsequently can lead to a broad distribution of element sizes. In explicit calculations, the critical time step $\Delta t_{\mathrm{c}}$ for stability scales with the size of the smallest element, which can result in steps much smaller than required to integrate the coarse sections of the mesh. Under these circumstances, Belytschko's subcycling algorithm, ${ }^{36-38}$ by permitting each element to be updated according to its own critical time step, can afford considerable speed-ups. In our implementation of the subcycling algorithm, element $e$ in the mesh is assigned a time step $\Delta t_{e}=m_{e} \Delta t_{\mathrm{c}}$, where $m_{e}$ is the largest integer such that $\Delta t_{e}$ does not exceed the stability limit of the element. Each node $a$ is then assigned a time step $\Delta t_{a}$ equal to the largest step $\Delta t_{e}$ among all elements connected to it. Counters are kept for each element and node to determine when an update is necessary. When an element is updated, displacements at nodes on adjacent slower elements are advanced by a linear interpolation in time. This strategy has been shown to result in the least noise. ${ }^{37}$ Details of the algorithm are given in Box 1. 
Box 1. Subcycling algorithm

(i) Initialize counters: $n_{e}=m_{e}, n_{a}=0$

(ii) Loop over nodes:

$$
\begin{aligned}
& \text { do } a=1, \text { numnp } \\
& \text { if } n_{a}=1 \text { then } \\
& \mathbf{u}_{a}=\Delta t_{a} \mathbf{v}_{a, n}+\frac{1}{2} \Delta t_{a}^{2} \mathbf{a}_{a, n} \\
& n_{a}=m_{a} \\
& \text { else } \\
& n_{a}=n_{a}-1 \\
& \text { end if } \\
& \mathbf{d}_{a, n+1}=\mathbf{d}_{a, n}+\mathbf{u}_{a} / m_{a} \\
& \text { end do }
\end{aligned}
$$

(iii) Loop over elements:

do $e=1$, numel

if $n_{e}=1$ then

update element with $\Delta t=\Delta t_{e}$

compute internal forces $\mathbf{R}_{n+1}^{e, \text { int }}$

$$
n_{e}=m_{e}
$$

else

$$
\begin{aligned}
& \mathbf{R}_{n+1}^{e, \text { int }}=\mathbf{0} \\
& n_{e}=n_{e}-1
\end{aligned}
$$

end if

end do

(iv) Compute velocities and accelerations for cycled nodes:

do $a=1$, numnp

$$
\begin{aligned}
& \text { if } n_{a}=m_{a} \text { then } \\
& \qquad \mathbf{a}_{a, n+1}=\left(\mathbf{R}_{a, n+1}^{\mathrm{ext}}-\mathbf{R}_{a, n+1}^{\mathrm{int}}\right) / M_{a} \\
& \quad \mathbf{v}_{a, n+1}=\mathbf{v}_{a, n}+\frac{1}{2} \Delta t_{a}\left[\mathbf{a}_{a, n}+\mathbf{a}_{a, n+1}\right] \\
& \text { end if } \\
& \text { end do }
\end{aligned}
$$

Subcycling is particularly effective for meshes containing elements of greatly disparate sizes. In the machining applications discussed subsequently, the cutting edge radius sets the scale for the element size near the tip of the tool, while the mesh size away from the tool typically scales with the depth of cut. In these applications we have found that subcycling speeds up the calculations by a factor in the range $2-3$.

\section{CONTACT}

Machining involves contact between deformable bodies, e.g., the workpiece and the tool. Meshon-mesh contact can also occur between the faces of cracks propagating though the chip and between detached segments. Here we use the predictor-corrector method developed by Taylor and Flanagan ${ }^{39}$ for the PRONTO2D explicit dynamics code. The bodies in contact can be deformable or rigid. Two contacting surfaces are designated as master and slave surfaces. The method starts by calculating the nodal accelerations from the out-of-balance forces and then 


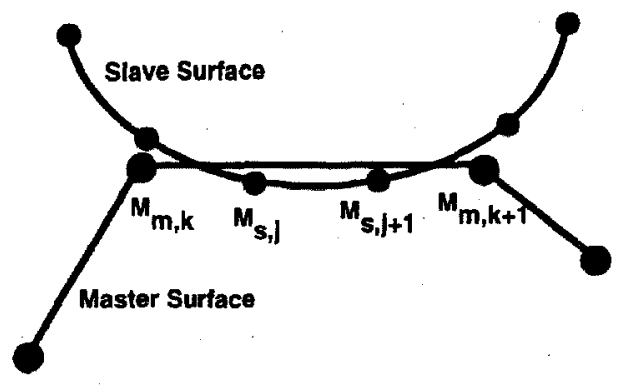

(a)

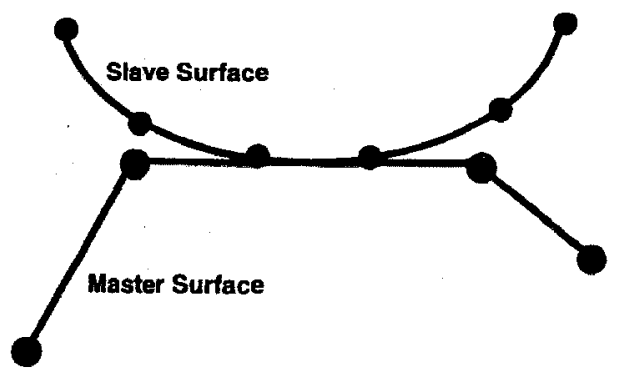

(b)

Figure 1(a) Predictor configuration of surfaces and (b) kinematically compatible surfaces

computing predictor nodal positions, velocities and accelerations $\mathbf{x}_{n+1}^{\text {pred }}, \mathbf{v}_{n+1}^{\text {pred }}$ and $\mathbf{a}_{n+1}^{\text {pred }}$, respectively, assuming that no contact has occurred. A resulting predictor configuration wherein penetration has occurred is shown in Figure 1(a).

In order to establish the contact conditions, an auxiliary consecutive numbering of the nodes on the contacting surfaces is introduced. The penetration distances $\delta_{s, j}$ for all nodes $j$ on the slave surface are then determined on the predictor configuration. Here and henceforth, labels $m$ and $s$ are used to designate the master and slave surfaces, respectively. The contact forces required to prevent penetration, were the master surface to remain stationary at the predictor configuration, are given by

$$
P_{\mathrm{s}, j}=\frac{M_{\mathrm{s}, j} \delta_{\mathrm{s}, j}}{\Delta t^{2}}
$$

where $M_{s, j}$ is the mass of node $j$ on the slave surface. Next, normal acceleration corrections are introduced which eliminate the unwanted penetration, Figure 1(b). The requisite accelerations are

$$
\begin{aligned}
& a_{m, k}^{\mathrm{corr}}=\frac{\sum_{j}\left(\omega_{\mathrm{s} \rightarrow m, j} P_{\mathrm{s}, j}\right)}{M_{m, k}+\sum_{j}\left(\omega_{\mathrm{s} \rightarrow m, j} M_{\mathrm{s}, j}\right)} \\
& a_{\mathrm{s}, j}^{\mathrm{corr}}=\sum_{k}\left(\omega_{m \rightarrow \mathrm{s}, k} a_{m, k}^{\mathrm{corr}}\right)-\frac{P_{\mathrm{s}, j}}{M_{\mathrm{s}, j}}
\end{aligned}
$$

where $\omega_{\mathrm{s} \rightarrow m, j}$ and $\omega_{m \rightarrow s, k}$ are weights dependent on position. A Coulomb friction model is also adopted from Taylor and Flanagan. ${ }^{40}$ Let $t$ represent the tangent to the master segment. The tangential component of the relative predictor velocity between the slave node and the master segment is given by

$$
\Delta v_{\mathrm{s}, j}=\mathbf{t} \cdot\left(\mathbf{v}_{\mathrm{s}, j}^{\text {pred }}-\sum_{k} \omega_{m \rightarrow s, k} \mathbf{v}_{m, k}^{\text {pred }}\right)
$$

The force that must be applied to the slave node to cancel its relative tangential velocity, i.e., to produce perfect stick, is

$$
F_{\mathrm{s}, j}^{\mathrm{stick}}=-\frac{M_{\mathrm{s}, j} \Delta v_{\mathrm{s}, j}}{\Delta t}
$$


The tangential force exerted by the master surface on a slave node cannot exceed the maximum frictional resistance

$$
F_{\mathrm{s}, j}=\frac{F_{\mathrm{s}, j}^{\text {stick }}}{\left|F_{\mathrm{s}, j}^{\text {stick }}\right|} \min \left(\mu N_{\mathrm{s}, j},\left|F_{\mathrm{s}, j}^{\text {stick }}\right|\right)
$$

where $N_{\mathrm{s}, j}$ is the normal contact force given by

$$
N_{\mathrm{s}, j}=M_{\mathrm{s}, j} a_{\mathrm{s}, j}^{\mathrm{corr}} \cdot \mathbf{n}
$$

where $\mathbf{n}$ is the surface normal. The tangential force generates the additional tangential acceleration corrections

$$
\begin{aligned}
& a_{\mathrm{s}, j}^{\mathrm{corr}}=\frac{F_{\mathrm{s}, j}}{M_{\mathrm{s}, j}} \\
& a_{m, k}^{\mathrm{corr}}=-\frac{\sum_{j} \omega_{\mathrm{s} \rightarrow m, j} F_{\mathrm{s}, j}}{M_{m, k}}
\end{aligned}
$$

A balanced master-slave approach in which surfaces alternately act as master and slave is employed however, rigid surfaces are always treated as master surfaces.

\section{THERMAL EFFECTS}

In applications such as machining, substantial amounts of heat may be generated due to the plastic working of the solid and friction at the tool-chip interface. The temperatures attained can be quite high and have a considerable influence on the mechanical response. The relevant balance law, in this case, is the first law, which can be expressed in weak form as

$$
\int_{B_{\mathrm{t}}} \rho c \dot{T} \eta \mathrm{d} V+\int_{\partial B_{t q}} h \eta \mathrm{d} S=\int_{B_{\mathrm{t}}} \mathbf{q} \cdot \nabla \eta \mathrm{d} V+\int_{B_{\mathrm{t}}} s \eta \mathrm{d} V
$$

where $\rho$ is the current mass density, $c$ the heat capacity, $T$ the spatial temperature field, $\eta$ an admissible virtual temperature field, $h$ the outward heat flux through the surface, $q$ is the heat flux, $s$ is the distributed heat source density, and $B_{t q}$ the current Neumann boundary. In machining applications, the main sources of heat are plastic deformation in the bulk and frictional sliding at the tool-workpiece interface. The rate of heat supply due to the first is estimated as

$$
s=\beta \dot{W}^{\mathfrak{p}}
$$

where $\dot{W}^{\mathrm{p}}$ is the plastic power per unit deformed volume and the Taylor-Quinney ${ }^{41}$ coefficient $\beta$ if of the order of 0.9 (e.g. Reference 42). The rate at which heat is generated at the frictional contact, on the other hand is

$$
h=-\mathbf{t} \cdot \llbracket \mathbf{v} \rrbracket
$$

where $t$ is the contact traction and $\llbracket \mathbf{v} \rrbracket$ is the jump in velocity across the contact. This heat must be apportioned between the tool and chip. Using transient half-space solutions, the ratio of the heat supply to the chip, $h_{1}$, and the tool, $h_{2}$, can be computed as (cf. Reference 7)

$$
\frac{h_{1}}{h_{2}}=\frac{\sqrt{k_{1} \rho_{1} c_{1}}}{\sqrt{k_{2} \rho_{2} c_{2}}}
$$


where $k_{\alpha}, \rho_{\alpha}$ and $c_{\alpha}, \alpha=1,2$, are the thermal conductivity, mass density and heat capacity of the workpiece and the tool, respectively.

The solid is assumed to obey Fourier's law of heat conduction, however, due to the finite kinematics care must be exercised in formulating Fourier's law so as to satisfy material frame indiffernece. To this end, thermal conductivity is regarded as a property of the crystal lattice which, therefore, is unchanged by plastic deformation. On this basis, a constant conductivity tensor $\overline{\mathbf{D}}$ is introduced on the intermediate configuration $\bar{B}_{t}$. The spatial conductivity tensor then follows as

$$
\mathbf{D}=\mathbf{F}^{\mathrm{e}} \overline{\mathbf{D}} \overline{\mathbf{F}}^{\mathrm{eT}}
$$

and Fourier's law can be written in spatial form as

$$
\mathbf{q}=-\mathbf{D} \cdot \nabla \mathbf{T}
$$

If the lattice conductivity is isotropic, then $\overline{\mathbf{D}}=k \mathbf{I}$ and (25) reduces to

$$
\mathbf{D}=k \mathbf{B}^{\mathbf{e}}
$$

where $\mathbf{B}^{\mathbf{e}}$ is the elastic left Cauchy-Green deformation tensor.

Inserting the finite element interpolation into (21) results in the semi-discrete system of equations $^{32}$

$$
\mathbf{C} \mathbf{T}+\mathbf{K T}=\mathbf{Q}
$$

where $\mathbf{T}$ is the array of nodal temperatures,

$$
C_{a b}=\int_{B_{\boldsymbol{c}}} \rho c N_{a} N_{b} \mathrm{~d} V
$$

is the heat capacity matrix,

$$
K_{a b}=\int_{B_{t}} D_{i j} N_{a, i} N_{b, j} \mathrm{~d} V
$$

is the conductivity matrix, and

$$
Q_{a}=\int_{B_{t}} s N_{a} \mathrm{~d} V-\int_{\partial B_{\mathrm{ta}}} h_{\alpha} N_{a} \mathrm{~d} S
$$

is the heat source array with $h_{\alpha}, \alpha=1,2$ having the appropriate value for the chip or tool as in (24). However, heat conduction between contacting bodies is not taken into account. In the applications of interest here, the mechanical equations always set the critical time step for stability. It therefore suffices to inrtegrate the energy equation (28) by the forward Euler algorithm, ${ }^{30-32}$ with the result

$$
\begin{aligned}
& \mathbf{T}_{n+1}=\mathbf{T}_{n}+\Delta t \dot{\mathbf{T}}_{n} \\
& \mathbf{C} \dot{\mathbf{T}}_{n}+\mathbf{K}_{n} \mathbf{T}_{n}=\mathbf{Q}_{n}
\end{aligned}
$$

We use a lumped capacity matrix $\mathbf{C}$, which eliminates the need for any equation solving. Sub-cycling techniques for parabolic equations, ${ }^{36}$ are available which afford speed-ups comparable to those obtained for hyperbolic problems.

\section{THERMO-MECHANICAL COUPLING}

A staggered procedure ${ }^{43}$ is adopted for the purpose of coupling the thermal and mechanical equations. In our implementation, we allow for different meshes for the thermal and mechanical 
models, which exchange information by the mesh-transfer operator described in Section 9. Mechanical and thermal computations are staggered assuming constant temperature during the mechanical step and constant heat generation during thermal step. Following Lemonds and Needleman, ${ }^{44} \mathrm{~A}$ mechanical step is taken first based on the current distribution of temperatures, and the heat generated is computed from (22) and (23). The heat thus computed is transferred to the thermal mesh and the temperatures are recomputed by recourse to the forward-Euler algorithm (32) and (33). The resulting temperatures are transferred to the mechanical mesh and are incorporated into the thermal-softening model described in Section 7, which completes one time-stepping cycle. A schematic flowchart of the staggered procedure is given in Box 2.

Box 2. Staggered procedure for thermo-mechanical coupling

(i) Initialize $\mathbf{T}_{1}=\mathbf{T}_{0}+\Delta t \dot{\mathbf{T}}_{0}, n=0$.

(ii) Isothermal mechanical step:

$$
\left\{\mathbf{x}_{n}, \mathbf{v}_{n}, \mathbf{a}_{n}, \mathbf{T}_{n+1}\right\} \rightarrow\left\{\mathbf{x}_{n+1}, \mathbf{v}_{n+1}, \mathbf{a}_{n+1}, \mathbf{T}_{n+1}\right\}
$$

(iii) Heat generation (bulk + contact)

(iv) Rigid conductor step:

$$
\left\{\mathbf{x}_{n+1}, \mathbf{v}_{n+1}, \mathbf{a}_{n+1}, \mathbf{T}_{n+1}\right\} \rightarrow\left\{\mathbf{x}_{n+1}, \mathbf{v}_{n+1}, \mathbf{a}_{n+1}, \mathbf{T}_{n+2}\right\}
$$

(v) $n \rightarrow n+1$, GOTO (ii)

An alternative staggered procedure based on an 'adiabatic split' has been proposed by Armero and Simó with a view to ensuring unconditional stability in implicit calculations.

\section{CONSTITUTIVE MODEL AND STRESS-UPDATE ALGORITHM}

We adopt a standard formulation of finite deformation plasticity based on multiplicative kinematics. In addition, we effect the requisite constitutive updates by a fully implicit update algorithm proposed by Cuitiño and Ortiz. ${ }^{47}$ The governing constitutive equations and their discretized counterparts are collected in Box 3.

Box 3. Constitutive framework and state-update algorithm

$\begin{aligned} & \text { Constitutive: } \leftrightarrow \text { Incremental: } \\ & \mathbf{F}=\mathbf{F}^{\mathrm{e}} \mathbf{F}^{\mathrm{p}} \leftrightarrow \mathbf{F}_{n+1}=\mathbf{F}_{n+1}^{\mathrm{e}} \mathbf{F}_{n+1}^{\mathrm{p}} \\ & \dot{\mathbf{F}}^{\mathrm{p}} \mathbf{F}^{\mathrm{p}-1}=\dot{\varepsilon}^{\mathrm{p}} \overline{\mathbf{R}}(\overline{\mathbf{S}}, \overline{\mathbf{Q}}) \leftrightarrow \mathbf{F}_{n+1}^{\mathrm{p}}=\exp \left(\Delta \varepsilon^{\mathrm{p}} \overline{\mathbf{R}}_{n+1}\right) \mathbf{F}_{n}^{\mathrm{p}} \\ & \overline{\mathbf{S}}=\overline{\mathbf{S}}\left(\frac{1}{2} \log \mathbf{C}^{\mathrm{e}}\right) \leftrightarrow \overline{\mathbf{S}}_{n+1}=\overline{\mathbf{S}}\left(\frac{1}{2} \log \overline{\mathbf{C}}_{n+1}^{\mathrm{e}}\right) \\ & \dot{\overline{\mathbf{Q}}}=\dot{\varepsilon}^{\mathrm{p}} \overline{\mathbf{H}}(\overline{\mathbf{S}}, \overline{\mathbf{Q}}) \leftrightarrow \overline{\mathbf{Q}}_{n+1}=\overline{\mathbf{Q}}_{n}+\Delta \varepsilon^{\mathrm{p}} \overline{\mathbf{H}}_{n+1} \\ & \dot{\varepsilon}^{\mathrm{p}}=\frac{1}{\eta} \bar{\phi}(\overline{\mathbf{S}}, \overline{\mathbf{Q}}) \leftrightarrow \Delta \varepsilon^{\mathrm{p}}=\frac{\Delta t}{\eta} \bar{\phi}_{n+1}\end{aligned}$

We assume that the deformation gradients $\mathbf{F}$ admit a multiplicative decomposition into elastic and plastic parts, $\mathbf{F}^{\mathbf{e}}$ and $\mathbf{F}^{\mathbf{p}}$, respectively. The plastic part $\mathbf{F}^{\mathbf{p}}$ of the deformation gradient field 
defines an additional (generally incompatible) configuration $\bar{B}_{t}$, or intermediate configuration. An overbar is used to identify fields defined over the intermediate configuration. The plastic flow rule, as stated here, determines $\dot{\mathbf{F}}^{p}$ fully. The manner in which the flow rule is discretized is an essential part of the method, and consists of taking the plastic flow direction $\overline{\mathbf{R}}$ to be constant throughout the increment and equal to its final value $\overline{\mathbf{R}}_{n+1}$. This reduces th flow rule to a system of linear equations for $\mathbf{F}^{\mathrm{p}}$ with initial conditions $\mathbf{F}_{n}^{\mathrm{p}}$, the exact solution of which is given by the exponential mapping.

The elastic response is expressed in terms of logarithmic elastic strains $(1 / 2) \log \overline{\mathbf{C}}^{\mathrm{e}}$, where $\overline{\mathbf{C}}^{\mathrm{e}}=\mathbf{F}^{\mathrm{eT}} \mathbf{F}^{\mathrm{e}}$ is the elastic right Cauchy Green deformation tensor. The stress measure $\overline{\mathbf{S}}$ is the second Piola-Kirchhoff stress tensor relative to the intermediate configuration. The symbol $\overline{\mathbf{Q}}$ denotes some suitable set of internal variables referred to the intermediate configuration, with $\overline{\mathbf{H}}$ the corresponding hardening moduli. Finally, $\bar{\phi}$ is an effective overstress and $\eta$ a viscosity parameter. Note that, by expressing the flow rule, elastic response and hardening laws on the intermediate configuration, the formulation automatically statistics material frame indifference.

The constitutive relations in Box 3 define a system of nonlinear equations which, for given $\mathbf{F}_{n+1}$, can be solved for the updated state variables $\overline{\mathbf{S}}_{n+1}, \overline{\mathbf{Q}}_{n+1}, \overline{\mathbf{F}}_{n+1}^{\mathbf{p}}$, as well as for $\Delta \varepsilon^{\mathrm{p}}$. As noted by Cuitiño and Ortiz, ${ }^{26}$ it is possible to reduce the system to a single equation for $\Delta \varepsilon^{\mathrm{p}}$, which can be solved by recourse to a local Newton-Raphson iteration. Cuitiño and Ortiz ${ }^{47}$ have also noted that the update defined in the foregoing furnishes a material-independent extension of smallstrain updates into the finite deformation range. The full finite deformation update comprises three steps:

(i) Preprocessor step: Compute the predictor logarithmic elastic strains (1/2) $\log \overline{\mathbf{C}}_{n+1}^{\mathbf{e} *}$ from the given updated deformation gradients $\mathbf{F}_{n+1}$. Set the predictor small-strain tensor $\boldsymbol{\varepsilon}_{n+1}^{e *}$ equal to $(1 / 2) \log \overline{\mathbf{C}}_{n+1}^{e *}$. Identify the small-strain stress tensor $\boldsymbol{\sigma}_{n}$ and $\overline{\mathbf{S}}_{n}$ and the small-strain internal variables $\mathbf{q}_{n}$ with $\overline{\mathbf{Q}}_{n}$.

(ii) Small-strain update: Effect a small-strain update driven by $\varepsilon_{n+1}^{e *}$ with initial conditions $\boldsymbol{\sigma}_{n}$ and $\mathbf{q}_{n}$, to compute $\boldsymbol{\sigma}_{n+1}, \mathbf{q}_{n+1}$ and $\Delta \varepsilon^{\mathrm{p}}$.

(iii) Postprocessor step: Identify $\overline{\mathbf{S}}_{n+1}$ with $\boldsymbol{\sigma}_{n+1}$ and $\overline{\mathbf{Q}}_{n+1}$ with $\mathbf{q}_{n+1}$, and compute $\mathbf{F}_{n+1}^{\mathbf{p}}$ by the exponential mapping.

It should be noted that the steps preceding and following the small-strain update are purely kinematic in nature, and, hence, material independent. Thus, when it applies, the above procedure provides a material-independent prescription for extending small-strain updates into the finite deformation range within the framework of multiplicative plasticity.

The finite plasticity formulation just described has some points of contact with the work of Weber and Anand, ${ }^{48}$ such as the use of the exponential mapping for the implicit integration of the flow rule. Weber and Anand ${ }^{48}$ limit their discussion to the isotropic solid and provide a set of approximate tangents based on a Pade approximation of the logarithmic mapping. Eterovic and Bathe $^{49}$ extended the work of Weber and Anand ${ }^{48}$ by considering the case of combined kinematic-isotropic hardening, but did not addressed the problem of calculating the consistent tangents, which were given by Cuitiño and Ortiz ${ }^{47}$ in closed form. Finally, it bears emphasis that neither Weber and Anand ${ }^{48}$ nor Eterovic and Bathe $^{49}$ discuss the connection between finite deformation and small-strain constitutive relations and update algorithms.

In a typical high-speed machining event, very high strain rates in excess of $10^{5} \mathrm{~s}^{-1}$ may be attained within the primary shear zone, while the remainder of the workpiece deforms at moderate or low strain rates. Under these conditions, a power viscosity law with constant rate sensitivity $m$ is not adequate. Indeed, the experimental stress-strain rate curves ${ }^{40,50,51}$ for structural steels exhibit a transition at strain rates of the order of $10^{5}-10^{6} \mathrm{~s}^{-1}$ from low to high 
rate sensitivity. At low strain rates, a rate sensitivity exponent in the range 100-200 adequately fits the data, while in the high strain rate regime a much lower rate sensitivity exponent in the range 5-20 applies. Modelling this type of behaviour is important, since a high rate sensitivity in the primary shear zone may lead to an elevation in stress which in turn can promote brittle fracture. A simple model which accounts for this behaviour consists of assuming a stepwise variation of the rate sensitivity exponent $m$ while maintaining continuity of stress. This leads to the relation

$$
\begin{aligned}
\left(1+\frac{\dot{\varepsilon}^{\mathrm{p}}}{\dot{\varepsilon}_{0}^{\mathrm{p}}}\right) & =\left(\frac{\bar{\sigma}}{g\left(\varepsilon^{\mathrm{p}}\right)}\right)^{m_{1}} \text { if } \dot{\varepsilon}^{\mathrm{p}} \leqslant \dot{\varepsilon}_{\mathrm{t}} \\
\left(1+\frac{\dot{\varepsilon}^{\mathrm{p}}}{\dot{\varepsilon}_{0}^{\mathrm{p}}}\right)\left(1+\frac{\dot{\varepsilon}_{t}}{\dot{\varepsilon}_{0}^{\mathrm{p}}}\right)^{m_{2} / m_{1}-1} & =\left(\frac{\bar{\sigma}}{g\left(\varepsilon^{\mathrm{p}}\right)}\right)^{m_{2}} \quad \text { if } \dot{\varepsilon}^{\mathrm{p}}>\dot{\varepsilon}_{\mathrm{t}}
\end{aligned}
$$

where $\bar{\sigma}$ is the effective Mises stress, $g$ the flow stress, $\varepsilon^{\mathbf{p}}$ the accumulated plastic strain, $\dot{\varepsilon}_{0}^{p}$ a reference plastic strain rate, $m_{1}$ and $m_{2}$ are low and high strain rate sensitivity exponents, respectively, and $\dot{e}_{\mathrm{t}}$ is the threshold strain rate which separates the two regimes. In calculations, we begin by computing $\dot{\varepsilon}^{\text {p }}$ according to (34), and switch to (35) if the result lies above $\dot{\varepsilon}_{\mathrm{t}}$.

Following Lemonds and Needleman, ${ }^{44}$ we also adopt a power hardening law with linear thermal softening. This gives

$$
g\left[1-\alpha\left(T-T_{0}\right)\right] \sigma_{0}\left(1+\frac{\varepsilon^{\mathrm{p}}}{\varepsilon_{0}^{\mathrm{p}}}\right)^{1 / n}
$$

where $n$ is the hardening exponent, $T$ the current temperature, $T_{0}$ a reference temperature, $\alpha$ a softening coefficient, and $\sigma_{0}$ is the yield stress at $T_{0}$. It should be noted that, owing to the staggered integration of the coupled thermal-mechanical equations, the temperature $T$ remains fixed during a mechanical step and, therefore, plays the role of a known parameter during a stress update.

\section{FRACTURE CRITERIA}

The process of segmented and discontinuous chip formation involves the propagation of fractures through the deforming chip. The simulation of these chip morphologies therefore requires the formulation of suitable fracture criteria, in conjunction with numerical procedures for nucleating and propagating a crack through the mesh.

Structural steels can fracture in a brittle or ductile manner (see Reference 52 for a review). Brittle fracture, such as occurs below the transition temperature, proceeds by cleavage. It is generally recognized that, when slip-induced transgranular cleavage is the dominant mechanism, fracture of mild steels can be described in terms of a critical stress criterion. ${ }^{53}$ In particular, Ritchie et $a l .{ }^{53}$ found the conditions for mode I brittle fracture to be consistent with the attainment of a critical opening stress $\sigma_{\mathrm{f}}$ at a critical distance $l$, or RKR criterion. The critical stress $\sigma_{\mathrm{f}}$ appears to be relatively independent of temperature and strain, rate, ${ }^{54,55}$ and can be inferred from the toughness $K_{\mathrm{IC}}$ through the small-scale yielding relation

$$
\sigma_{\mathrm{f}}=\frac{K_{\mathrm{IC}}}{\sqrt{2 \pi l}}
$$

The critical distance $l$ correlates with the spacing the grain boundary carbides, typically of the order of two grain diameters. ${ }^{53}$

Under mixed-mode conditions, such as may be expected in machining, the crack may kink or follow a curved path as it grows. In order to predict the crack trajectory under conditions of 
brittle fracture, we adopt the maximum hoop stress criterion of Erdogan and Sih. ${ }^{56}$ According to this theory, the crack propagates along the angle $\theta$ from the crack face at which the hoop stress $\sigma_{\theta \theta}$ attains a relative maximum. The resulting trajectory of the crack is such that $K_{\mathrm{II}} \approx 0$ at the crack tip. Combining the maximum hoop stress and RKR criteria, the condition for mixed-mode crack growth is taken to be

$$
\max _{\theta} \sigma_{\theta \theta}(l, \theta)=\sigma_{\mathrm{f}}
$$

with the understanding that the crack propagates at the angle $\theta$ for which the criterion is met.

Void growth and coalescence is known to be a principal mechanism of ductile fracture in structural steels. ${ }^{57-60}$ Voids are nucleated as a result of fracture of decohesion of carbides and subsequently grow as the surrounding material strains plastically. The rate of growth of the voids is accelerated by the blunting of the crack tip, which has the effect of raising the level of hydrostatic stress at the location of the void. The final stages of coalescence may be aided by the development of sheets of smaller voids nucleated from precipitate particles or carbides. The experimental evidence shows that the fracture toughness of metals depends on the size and spacing of void nucleating second phase particles. ${ }^{59,61-63}$

An approximate analysis of the growth of a spherical void before a blunting crack was given by Rice and Johnson. ${ }^{64}$ The analysis was based on earlier results by Rice and Tracey ${ }^{65}$ pertaining to the growth of an isolated spherical void in an ideally plastic material. Rice and Johnson ${ }^{64}$ used the Rice and Tracey $^{65}$ solution with the remote fields identified with the local stress and deformation fields computed from a slip line solution of a void-free blunting crack. Rice and Johnson $^{64}$ were able to estimate values of the Crack Tip Opening Displacement (CTOD) for fracture initiation which were in good agreement with experiment. Following Ritchie $e t$ al., ${ }^{66}$ the critical CTOD criterion for mode I ductile fracture can be recast as the attainment of a critical value $\varepsilon_{\mathrm{f}}^{\mathrm{p}}$ of the effective plastic strain at a distance $l$ directly ahead of the crack tip. Proceeding as before, we express this criterion in the form

$$
\max _{\theta} \varepsilon^{\mathrm{p}}(l, \theta)=\varepsilon_{\mathrm{f}}^{\mathrm{p}}
$$

with the understanding that the crack propagates at the angle $\theta$ for which the criterion is met. Based on the Rice and Tracey solution, ${ }^{65}$ the critical effective plastic strain can be estimated as ${ }^{52}$

$$
\varepsilon_{\mathrm{f}}^{\mathbf{p}} \approx 2 \cdot 48 \mathrm{e}^{-1 \cdot 5 p / \bar{\sigma}}
$$

where $p=\sigma_{k k} / 3$ is the hydrostatic pressure ( $p>0$ for hydrostatic tension). The strong dependence of the critical effective plastic strain $\varepsilon_{\mathrm{f}}^{\mathrm{p}}$ on the triaxiality ratio $p / \bar{\sigma}$ is apparent from (40).

\section{ADAPTIVE MESHING}

One difficulty that arises in applying Lagrangian formulations to problems involving unconstrained plastic flow, such as machining, is that the mesh may become severely distorted. One way of sidestepping this difficulty, thereby extending the range of applicability of Lagrangian methods, is to resort to continuous remeshing. In this approach, the connectivity of the finite element mesh is redefined at regular intervals by triangulating the nodes at their spatial locations. This process of continuous remeshing, by itself, is capable of eliminating the bulk of the deformation-induced element distortion. Further gains can be achieved through the use of simple mesh smoothing algorithms. Besides sidestepping the problem of mesh distortion, adaptive meshing furnishes 
a means of simultaneously resolving multiple scales in the solution. Examples of fine features which drive mesh refinement in machining are the mechanical and thermal boundary layers which develop in the contact region and within localized shear bands. We also resort to a remeshing technique to propagate cracks through the mesh, as discussed in Section 10.

The adaptive meshing methodology used here is taken from Ortiz and Quigley, ${ }^{67}$ with a number of modifications to suit the application addressed here. All meshes are constructed by Delaunay triangulation ${ }^{68}$ and are, therefore, constrained to consist of triangular elements. The most commonly employed elements of this type are the first- and second-order isoparametric elements. Mixed elements possessing additional pressure degrees of freedom were not considered in view of the difficulties their use presents in the context of explicit dynamics. It is well-known that the first-order simplex suffers from volumetric and shear locking which can result in gross inaccuracies. $^{35,69}$ The elements have to be meshed in a cross-triangle configuration to give acceptable results. However, this configuration is inappropriate for the unstructured meshes we consider here. By contrast, the second-order element, which is our element of choice, has higher accuracy and does not lock. ${ }^{30}$

The connectivity of the mesh is determined from the set of corner nodes of the elements, with the mid-nodes added subsequently. For the applications discussed here, an adaptation criterion based on the equidistribution of plastic power has proved useful. In this approach, elements with plastic power contents exceeding a prescribed tolerance TOL, i.e., elements such that

$$
\int_{\Omega_{h}^{\mathrm{c}}} \dot{W}^{\mathrm{p}} \mathrm{d} \Omega>\mathrm{TOL}
$$

are targetted for refinement. Here, $\Omega_{h}^{\mathrm{e}}$ denotes the domain of element $e$, and, for the Mises solid, the plastic power density is given by

$$
\dot{W}^{\mathbf{p}}=\bar{\sigma} \dot{\varepsilon}^{\mathbf{p}}
$$

Clearly, this criterion leads to refinement in regions of high rate of plastic deformation. The plastic power equidistribution criterion is amenable to an error minimization interpretation in some simple model problems. ${ }^{70}$

The mesh is adapted at regular intervals by adding new corner nodes at the mid-sides of elements targetted for refinement. The element connectivity is then completely redefined by a Delaunay triangulation based on the new set of corner nodes. In particular, no hierarchical compatibility between subsequent meshes is enforced. Triangulations are effected on the deformed configuration, which contributes to eliminating much of the mesh distortion introduced by the flow of material. If, despite this continuous remeshing, elements arise with unacceptable aspect ratios, the mesh is subjected to laplacian smoothing. In addition, to prevent the deterioration of the isoparametric mapping, an update of the reference configuration is effected at regular intervals. Along with adaptive refinement, a mesh-coarsening algorithm is used in areas of the solid which have become inactive. In machining applications, mesh coarsening contributes to keeping the size of the problem within manageable bounds.

Finally, a transfer operator proposed by Ortiz and Quigley $^{67}$ is used to transfer the state variables between meshes following an adaptation. As shown by Ortiz and Quigley, ${ }^{67}$ the $\mathrm{Hu}$-Washizu variational principle provides a prescription for constructing state-transfer operators once the interpolation of the state variables is defined. We have found it effective to interpolate the state variables using an auxiliary triangular mesh in which the Gauss points of the elements supply the nodes. This has the effect of smoothing out local spikes in the state fields, such as inevitably arise near the tip of the tool, which would otherwise corrupt the transferred fields. In 


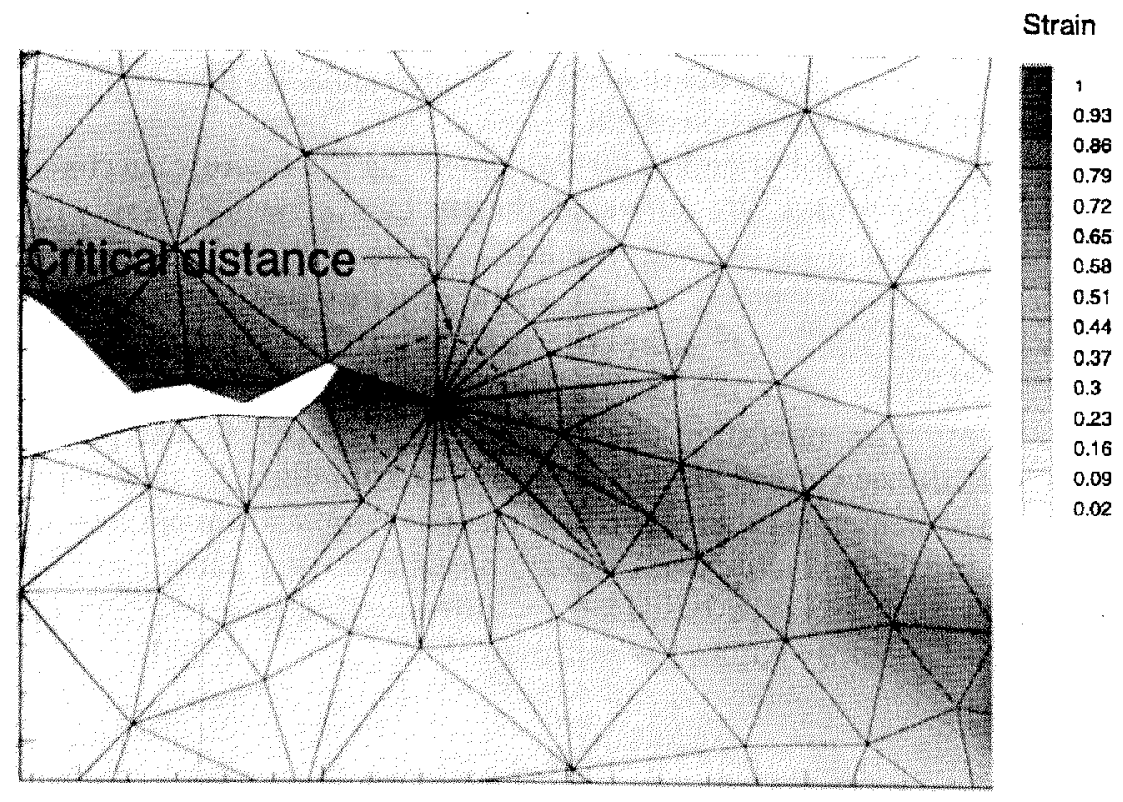

(a)

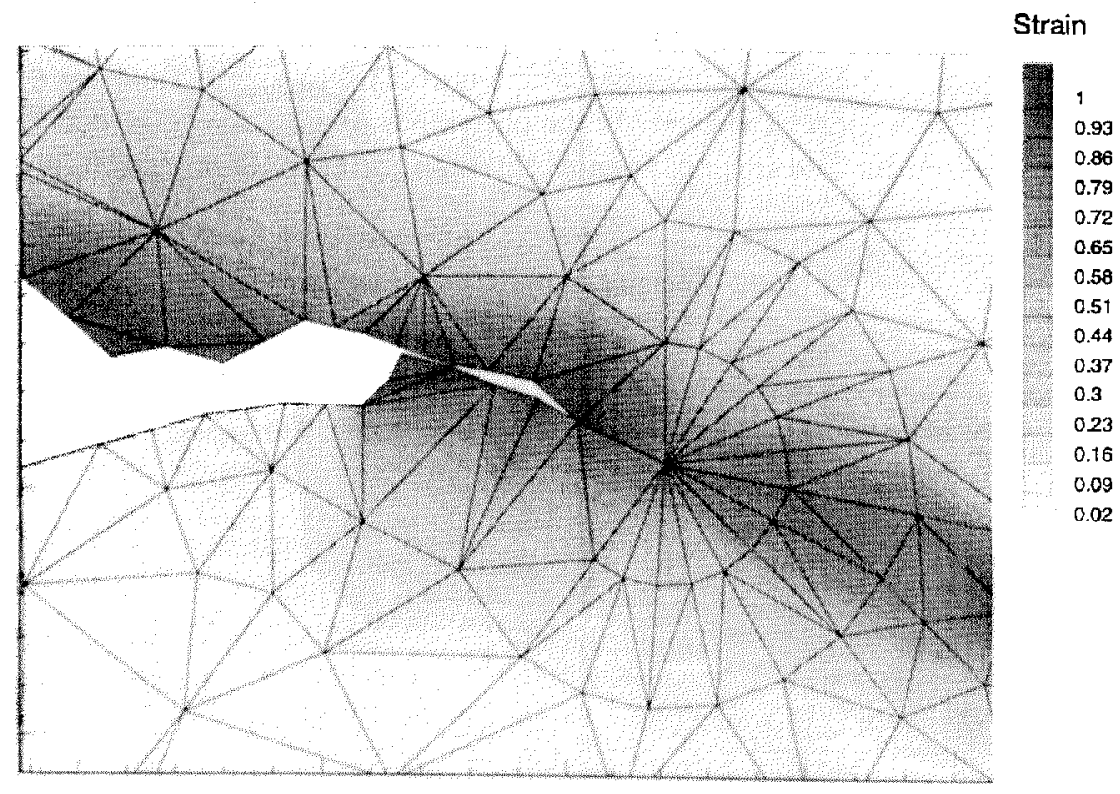

(b)

Figure 2. Procedure for advancing a crack tip: (a) initial configuration; (b) propagated configuration 
order to preserve the deformation jacobian, logarithmic strains and rotation tensors are transferred instead of the deformation gradients themselves. Constraints are also applied to the data to render the deformation and stresses consistent.

Mesh adaption is also our tool of choice for implementing the fracture mechanical aspects of the model discussed in Section 8. We surround the tip of a growing crack with a rosette of elements which provide the requisite angular resolution of the near-tip fields, Figure 2(a). The size of the elements in the rosette is chosen so that a row of gauss points is located at the critical sampling distance $l$ from the tip. The fracture criteria (39) and (38) are then checked at each of the critical gauss points. When one of the criteria is met, a new crack segment is placed in the corresponding direction. This operation entails doubling the node at the previous crack tip, placing a new node at the new crack tip location, and surrounding the new crack tip with the corresponding rosette of elements, Figure 2(b). By a recursive application of these operations over successive time steps, brittle or ductile crack growth along arbitrary paths can be simulated. An alternative method of crack advance based on the introduction of a cohesive law at element interfaces has been developed by $\mathrm{Xu}$ and Needleman. ${ }^{71}$

\section{APPLICATION}

The first application concerns the cutting of a rectangular block of high strength AISI 4340 steel at a velocity of $30 \mathrm{~m} \mathrm{~s}^{-1}$, with the tool modelled as rigid. The material and process parameters are collected in Tables I-III. The thermal properties of the tool are supposed to be matched to those of the workpiece, giving an equal proportion of frictional heat allotted to the tool and the chip.

At positive rake angles, the deformation is largely confined to the primary shear zone and to a boundary layer adjacent to the tool, as expected, Figure 3. No shear localization occurs and a continuous chip morphology is predicted. As the chip curls around and contacts the workpiece a crack initiates on the free surface and propagates through the chip thickness, finally severing the

Table I. Mechanical material constants

\begin{tabular}{lccccccccc}
\hline$E$ & $v$ & $\sigma_{y}$ & $m_{1}$ & $m_{2}$ & $\dot{\varepsilon}_{0}^{\mathrm{p}}$ & $\dot{\varepsilon}_{t}^{\mathrm{p}}$ & $K_{\mathrm{IC}}$ & $\mu$ & $\alpha$ \\
\hline $200 \mathrm{GPa}$ & $0 \cdot 3$ & $1090 \mathrm{MPa}$ & 100 & 5 & $1 \mathrm{~s}^{-1}$ & $2 \times 10^{5} \mathrm{~s}^{-1}$ & $30 \mathrm{MPa} \sqrt{m}$ & 0.25 & 0.001 per $^{\circ} \mathrm{C}$ \\
\hline
\end{tabular}

Table II. Thermal material constants

\begin{tabular}{lcc}
\hline Density & Conductivity & Heat capacity \\
\hline $7800 \mathrm{~kg} \mathrm{~m}^{-3}$ & $43 \mathrm{~W} \mathrm{~m}^{-1}$ per ${ }^{\circ} \mathrm{C}$ & $600 \mathrm{~J} / \mathrm{kg}^{-1}$ per ${ }^{\prime} \mathrm{C}$ \\
\hline
\end{tabular}

Table III. Machining simulations

\begin{tabular}{lcccc}
\hline Simulation & $\begin{array}{c}\text { Speed } \\
\left(\mathrm{m} \mathrm{s}^{-1}\right)\end{array}$ & $\begin{array}{c}\text { Rake angle } \\
(\mathrm{deg})\end{array}$ & $\begin{array}{c}\text { Depth of cut } \\
(\mu \mathrm{m})\end{array}$ & $\begin{array}{c}\text { Cutting edge } \\
\text { radius }(\mu \mathrm{m})\end{array}$ \\
\hline 1 & 30 & 10 & 250 & 25 \\
2 & 10 & -5 & 500 & 25 \\
3 & 20 & 5 & 500 & 25 \\
\hline
\end{tabular}



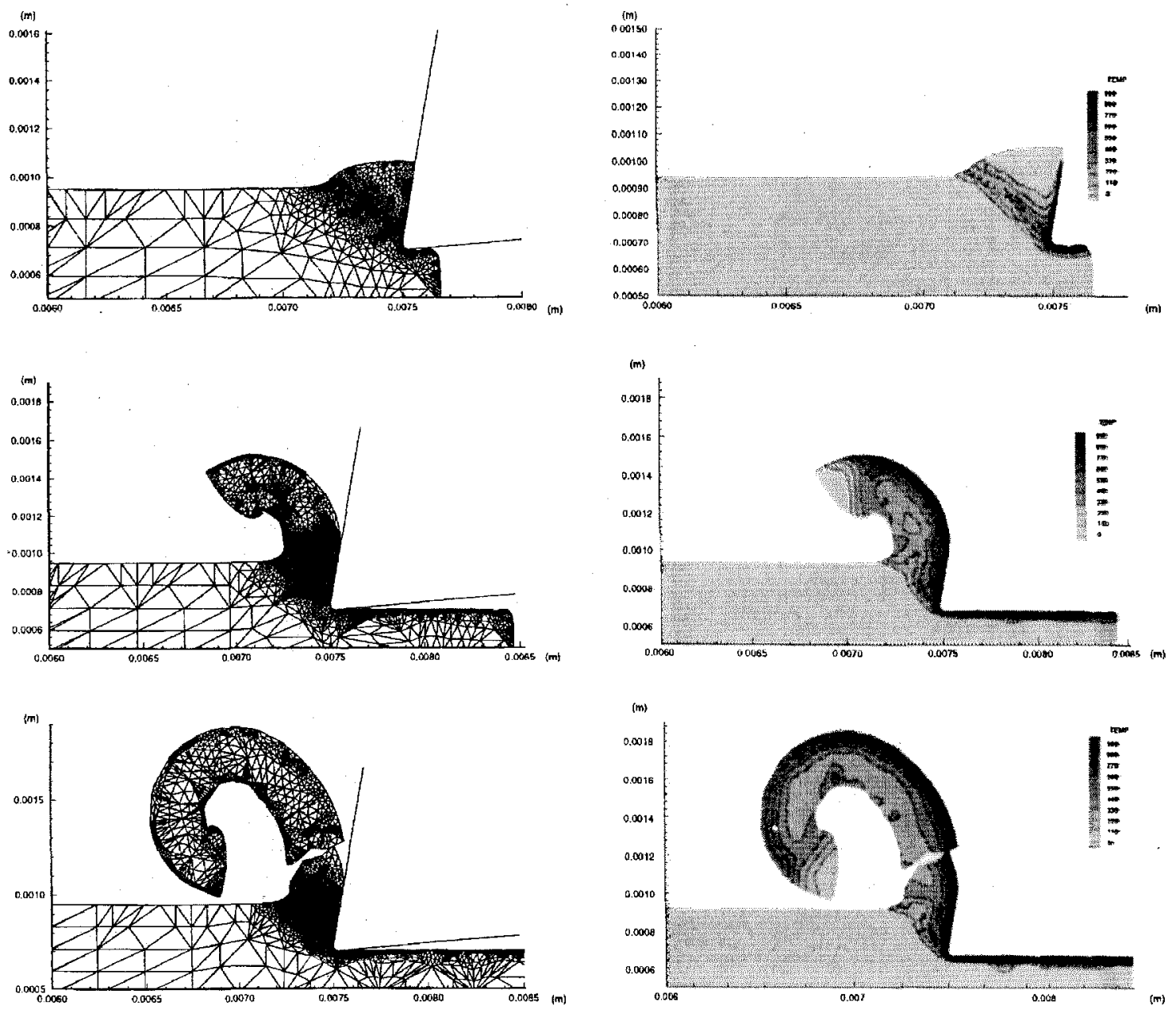

(a)

(b)

Figure 3. Continuous chip formation: (a) mesh; (b) temperature contours

chip. The extensive mesh refinement required to adequately resolve the solution is evident from Figure 3. The coarsening of the inactive parts of the mesh is also noteworthy. The maximum number of nodes in the mesh is near 2600 with about 1100 elements. Time steps were on the order of $3 \times 10^{-10} \mathrm{~s}$ which necessitated about $1 \times 10^{6}$ steps or $20 \mathrm{~h}$ or CPU time on a DEC 3000 workstation.

Average steady-state temperatures along the tool-chip interface are near $1000^{\circ} \mathrm{C}$ and drop off rapidly into the interior, Figure 3 . Due to the high cutting speed, heat conduction is of little consequence and conditions are nearly adiabatic. Frictional contact, as opposed to plastic dissipation in the bulk, accounts for most of the heat generated. The largest accumulated plastic strains occur within the boundary layer adjacent to the tool. In this region, the flow of material is facilitated by thermal softening and the plastic strains attain values of the order of 12 . Strains in the chip interior remain within the 1-2 range upon exit from the primary shear zone, and within 3-5 in the wake of the tool on the workpiece surface.

An inspection of the machined surface reveals surface waviness on the scale of the cutting edge radius. The material which flows under the tool tip experiences large compressive stresses 

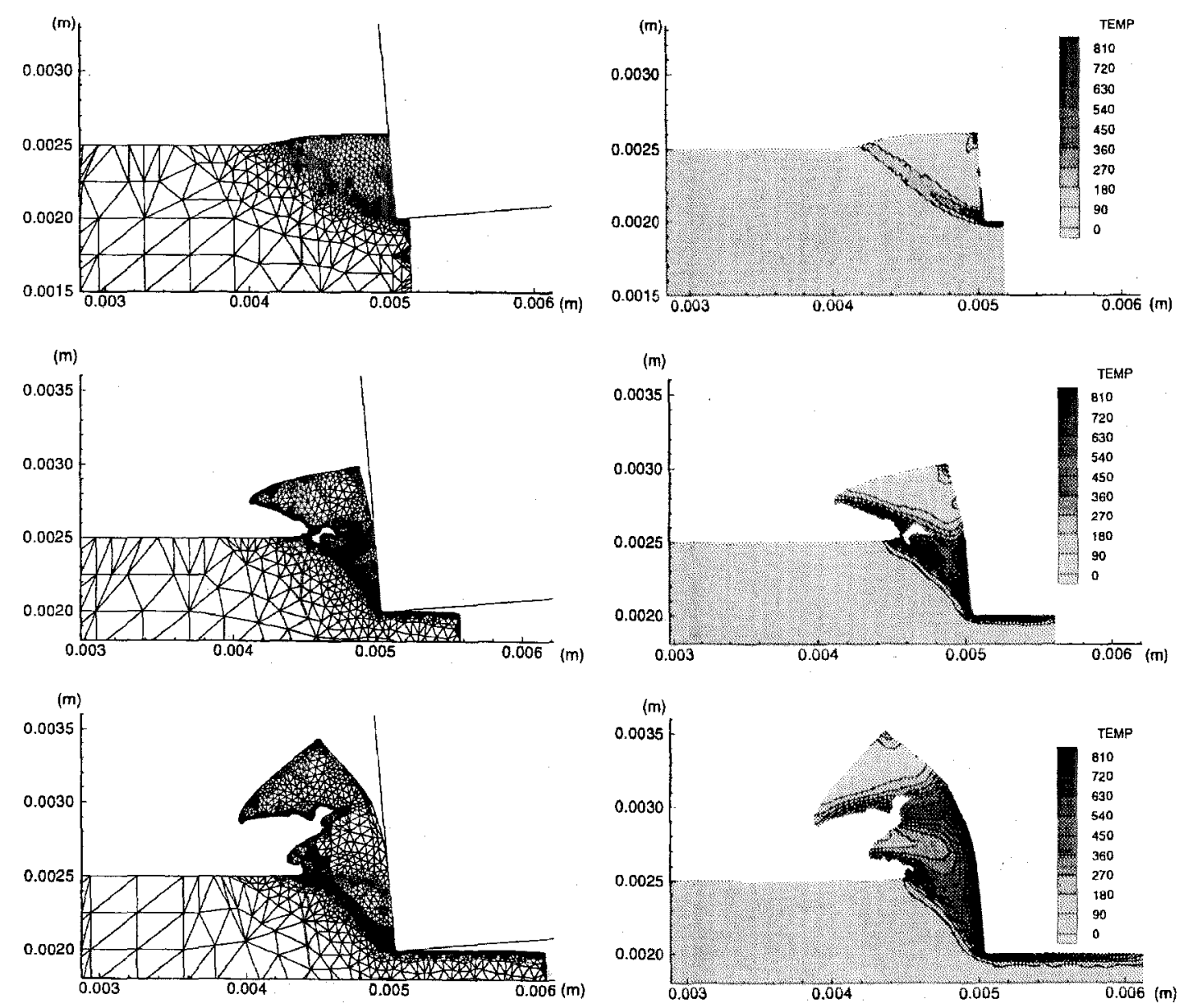

(a)

(b)

Figure 4. Shear localized chip formation: (a) mesh; (b) temperature contours

followed by rapid elastic unloading. This unloading may account for some of the surface roughness. We remark that the Lagrangian character of the simulation and the elastic-plastic constitutive description adopted enable the computation of the state of residual stress on the cut surface. Surface roughness and the state of residual stress under the surface may have a significant effect of the fatigue life of the component and, consequently, are of engineering interest.

Our second application concerns an AISI 4340 steel cut with a negative rake angle at a speed of $10 \mathrm{~m} \mathrm{~s}^{-1}$. The parameters used in the simulation are collected in Tables I-III. Following an initial transient similar to that observed in the preceding simulation, shear localization occurs along the primary shear band as the material softens thermally, Figure 4 . The shear band starts out straight but eventually bows out in a concave down fashion, a process which is accompanied by the initiation of a ductile crack at the surface of the chip. Fracture proceeds along the band and is promoted by rapid thermal softening. The crack propagates more than half the way through the chip thickness before arresting, thus completing the first segment of a shear-localized chip. Subequently, the primary shear band is swept downstream and its temperature profile diffuses out. This is followed by the formation of a new active shear band and the initiation of a ductile 

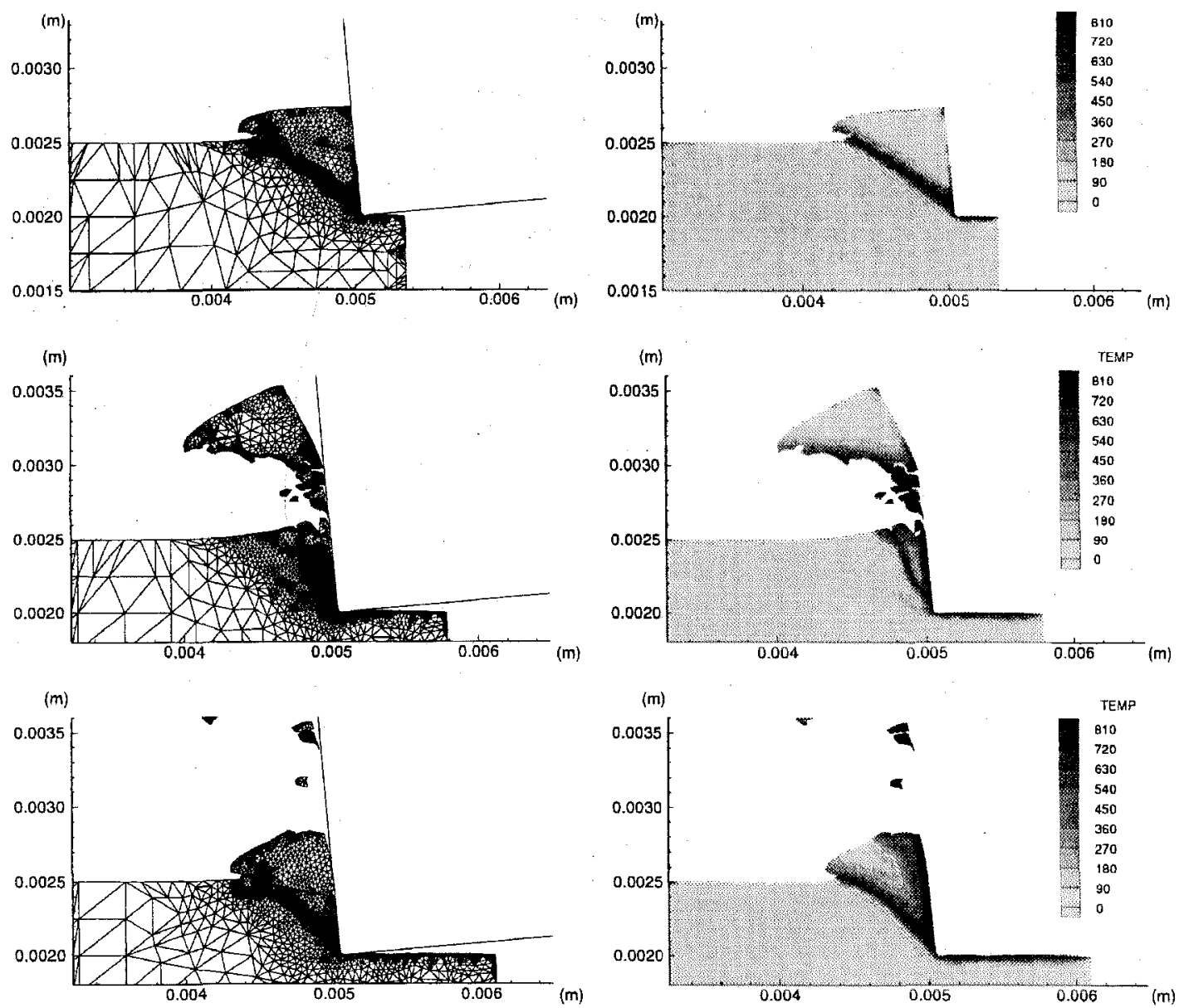

(a)

(b)

Figure 5. Discontinuous chip formation: (a) mesh; (b) temperature contours

crack at the surface, eventually leading to a second segment. The sequence of events repeats itself indefinitely a results in a 'shear localized' chip morphology, in agreement with observation. ${ }^{29}$ In other simulations performed the variation in mesh size, which directly affects the time step, had little influence on the location of initiated cracks.

The effective plastic strain attains values of the range 2-3 within the localized zone and 6-12 along the tool face. As in the preceding application, a high degree of mesh refinement is required to adequately resolve the fine features of the solution. It should be noted that the faces of the cracks which propagate along the primary shear zone often come in contact, which demonstrates the need for mesh-on-mesh contact capability even when the tool is idealized as rigid. The high plastic strains adjacent to the contact owe partly to the high temperatures which develop in that region, of the order of $990^{\circ} \mathrm{C}$, which result in considerable thermal softening. Temperature profiles within the primary shear band show peaks of $500^{\circ} \mathrm{C}$.

The final example concerns a shear localized chip in which the segments become completely detached, Figure 5 . The tool geometry is as in the preceding simulation and the cutting velocity is $20 \mathrm{~m} \mathrm{~s}^{-1}$. The initial segment forms as before but is now followed by a crack propagates through 
the entire thickness of the chip, causing the chip to become fully detached. Komanduri et al. ${ }^{29}$ have shown that the transitional speed for the onset of segment detachment is near $15 \mathrm{~m} \mathrm{~s}^{-1}$ for the material at hand, in agreement with our simulations. The strain rates in the primary shear band range between $3 \times 10^{5}-4.5 \times 10^{5} \mathrm{~s}^{-1}$. By contrast, the strain rates which arise in the same region for a cutting speed of $10 \mathrm{~m} \mathrm{~s}^{-1}$ are in the lower range of $1.5 \times 10^{5}-2 \times 10^{5} \mathrm{~s}^{-1}$. The elevated stresses which cause a through-thickness crack at the higher speed are furnished by the variable rate sensitivity model with the threshold strain rate set at $2 \times 10^{5} \mathrm{~s}^{-1}$, which is in the ballpark of experimental observation. ${ }^{40,50,51}$ Evidently, this threshold separates the strain rate ranges for the cutting velocities of 10 and $20 \mathrm{~m} \mathrm{~s}^{-1}$ considered, ensuring, that the transition to segmented chips takes place between those speeds.

\section{CONCLUSIONS}

We have assembled several numerical techniques which, in combination, enable the simulation of orthogonal high-speed machining. Finite deformation plasticity with thermal softening, explicit dynamics, mesh-on-mesh contact with friction, fully coupled heat conduction, continuous remeshing and fracture are the main elements of the model. Our simulations demonstrate the ability of the model to predict chip morphologies consistent with obsevation, perhaps the most severe test of any machining model. Detailed parametric studies are presently being conducted and will be reported in a forthcoming publication.

\section{ACKNOWLEDGEMENTS}

This work has been funded by the National Science Foundation through Grant DDM-9016568. The authors are indebted to Steve Wayne of the Valenite Advanced Technology Center for helpful suggestions and discussions.

\section{REFERENCES}

1. G. S. Vasilash, 'Cutting tools', Prod. Eng. Mag., 40-44 (May, 1992).

2. A. O. Tay, M. G. Stevenson, G. de Vahl Davis and P. L. Oxley, 'A numerical method for calculating temperature distributions in machining from force and shear angle measurements', Int. J. Mach. Tool Des. Res., 16, 335-349 (1976).

3. A. O. Tay, M. G. Stevenson and G. de Vahl Davis, 'Using the finite element method to determine temperature distributions in orthogonal machining', Proc. Instn. Mech. Engrs., 188, 627-638 (1974).

4. J. Lin, S. L. Lee and C. I. Weng, 'Estimation of cutting temperature in high speed machining', J. Eng. Mater. Technol., 114, 289-295 (1992).

5. J. S. Strenkowski and J. T. Carroll III, 'A finite element model of orthogonal metal cutting', J. Eng. Ind., 107, 349-354 (1985).

6. K. Komvopoulos and S. A. Erpenbeck, 'Finite element modeling of orthogonal metal cutting', J. Eng. Ind., 113, 253-267 (1991).

7. G. S. Sekhon and J. L. Chenot, 'Numerical simulation of continuous chip formation during non-steady orthogonal cutting', Eng. Comput., 10, 31-48 (1993).

8. S. Kalpakjian, 'Manfacturing Processes for Engineering Materials', Addison-Wesley, Reading, MA, (1984).

9. T. H. H. Pian and K. Sumihara, 'Rational approach for assumed stress finite elements', Int. j. numer. methods eng., 20, 1685-1695 (1984).

10. T. Belytschko and W. E. Bachrach, 'Efficient implementation of quadrilaterals with high coarse-mesh accuracy', Comput. Methods Appl. Mech. Eng., 54, 279-301 (1986).

11. E. N. Dvorkin and A. P. Vassolo, 'A quadrilateral 2D finite element based on mixed interpolation of tensorial components', Eng. Comput., 6, 217-224 (1989).

12. J. C. Simó and M. S. Rifai, 'A class of assumed strain methods and the method of incompatible modes', Int. j. numer. methods eng., 29, 1595-1638 (1990).

13. T. Belytschko and L. P. Bindeman, 'Assumed strain stabilization of the 4-node quadrilateral with 1-point quadrature for nonlinear problems', Comput. Methods Appl. Mech. Eng., 88, 311-340 (1991). 
14. J. C. Simó and F. Armero, 'Geometrically nonlinear enhanced strain mixed methods and the method of incompatible modes', Int. j. numer. methods eng., 33, 1413-1449 (1992).

15. J. C. Simó, F. Armero and R. L. Taylor, 'Improved versions of assumed enhanced strain tri-linear elements for 3D finite deformation problems', Comput. Methods Appl. Mech. Eng. (to appear).

16. T. J. R. Hughes, W. K. Liu and T. K. Zimmerman, 'Lagrangian-Eulerian finite element formulation for incompressible viscous flows', Comput. Methods Appl. Mech. Eng., 29, 329-349 (1981).

17. J. Donea, 'Arbitrary Lagrangian-Eulerian finite element methods', in T. Belytschko and T. J. R. Hughes (eds.), Computational Methods for Transient Analysis, North-Holland, New York, 1983, pp. 473-516.

18. W. K. Liu, T. Belytschko and H. Chang, 'An Arbitrary Lagrangian-Eulerian finite element method for pathdependent materials', Comput. Methods Appl. Mech. Eng., 58, 227-245 (1986).

19. W. K. Liu, H. Chang, J. S. Chen and T. Belytschko, 'Arbitrary Lagrangian-Eulerian Petrov-Galerkin finite elements for nonlinear continua', Comput. Methods Appl. Mech. Eng., 68, 259-310 (1988).

20. D. J. Benson, 'An efficient, accurate, simple ALE method for nonlinear finite element programs', Comput. Methods Appl. Mech. Eng., 72, 305-350 (1989).

21. S. Ghosh and N. Kikuchi, 'An arbitrary Lagrangian-Eulerian finite element method for large deformation analysis of elastic-viscoplastic solids', Comput. Method Appl. Mech. Eng., 86, 127-188 (1991).

22. D. J. Benson, 'Computational methods in Lagrangian and Eulerian hydrocodes', Comput. Methods Appl. Mech. Eng., 99, 235-394 (1992).

23. E. Thompson, 'Use of the pseudo-concentrations to follow creeping viscous flows during transient analysis', $J$. mumer. methods fluids, 6, 749-761 (1986).

24. E. Thompson and R. E. Smelser, 'Transient analysis of forging operations by the pseudo-concentrations methods', Int. j. numer. methods eng., 23, 177-189 (1988).

25. E. N. Dvorkin and E. G. Petöcz, 'On the modelling of $2 \mathrm{D}$ metal forming processes using the flow formulation and the pseudo-concentration technique', in D. R. J. Owen, E. Oñate and E. Hinton (eds.), Proc. 3rd Int. Conf. Comput. Plasticity, Pineridge Press Swansea, U.K., 1992, pp. 1037-1051.

26. P. K. Wright and E. M. Trent, 'Metallurgical appraisal of wear mechanisms and processes on high-speed-steel cutting tools', Metals Technol., 13-23 (1974).

27. S. Ramalingam and J. T. Black, 'An electron microscopy study of chip formation', Metall. Trans., 4, 1103-1112 (1973).

28. R. Komanduri and R. H. Brown, 'On the mechanics of chip segmentation in machining', J. Eng. Ind., 103, 33-42 (1981).

29. R. Komanduri, T. Schoeder, J. Hazra, B. F. von Turkovich and D. G. Flom, 'On the catastrophic shear instability in high-speed machining of an AISI 4340 steel', J. Eng. Ind., 104, 121-131 (1982).

30. T. J. R. Hughes, 'The Finite Element Method', Prentice-Hall, Englewood Cliffs, N.J., 1987.

31. T. J. R. Hughes and T. Belytschko, 'A Précis of developments in computational methods for transient analysis', J. Appl. Mech., 50, 1033-1041 (1983).

32. T. Belytschko, 'An overview of semidiscretization and time integration procedures', in T. Belytschko and T. $J$. R. Hughes (eds.), Computational Methods for Transient Analysis, North-Holland Amsterdam, 1983, pp. 1-65.

33. T. J. R. Hughes, 'Analysis of transient algorithms with particular reference to stability behavior', in T. Belytschko and T. J. R. Hughes (eds.), Computational Methods for Transient Analysis, North-Holland Amsterdam 1983, pp 67-155.

34. K. K. Mathur, A. Needleman and V. Tvergaard, 'Dynamic 3D analysis of the charpy V-notch test', Modelling Simul. Mater. Sci. Eng., 1, 467-484 (1993).

35. N. Rebelo, J. C. Nagtegaal, L. M. Taylor and R. Passman, 'Industrial application of implicit and explicit finite element methods to forming processes', in AMD-Vol. 156, Numerical Methods for Simulation of Industrial Metal Forming Processes, ASME, New York, 1992.

36. T. Belytschko, P. Smolinski and W. K. Liu, 'Stability of Multi-Time Step Partitioned Integrators for First-Order Finite Element Systems', Comput. Methods Appl. Mech. Eng., 49, 181-197 (1985).

37. T. Belytschko, H.-J. Yen and R. Mullen, 'Mixed methods for time integration', Comput. Methods Appl. Mech. Eng., 17/18, 259-275 (1989).

38. T. Belytschko, 'On computational methods for crashworthiness', Comput. Struct., 42, 271-279 (1992).

39. L. M. Taylor and D. P. Flanagan, 'PRONTO 2D: a two-dimensional transient solid dynamics program', Sandia National Laboratories, SAND86-0594, 1987.

40. R. W. Klopp, R. J. Clifton and T. G. Shawki, 'Pressure-shear impact and the dynamic viscoplastic response of metals', Mech. Mater., 4, 375-385 (1985).

41. G. I. Taylor and H. Quinney, 'The plastic distortion of metals', Philos. Trans. Roy. Soc. London, A230, 323-362 (1931).

42. S. Kobayashi, S.-I. Oh and T. Altan, 'Metal Forming and the Finite Element Method', Oxford University Press, Oxford (1989).

43. K. C. Park and C. A. Felippa, 'Partitioned analysis of coupled systems', in T. Belytschko and T. J. R. Hughes (eds.), Computational Methods for Transient Analysis, North-Holland, Amsterdam 1983, pp. 157-219.

44. J. Lemonds and A. Needleman, 'Finite element analysis of shear localization in rate and temperature dependent solids', Mech. Mater., 5, 339-361 (1986).

45. F. Armero and J. C. Simó, 'Algorithms for static and dynamic multiplicative plasticity that preserve the classical return mapping schemes of the infinitesimal theory', Comput. Methods Appl. Mech. Eng., 99, 61 (1992).

46. F. Armero and J. C. Simó, 'A-priori stability estimates and unconditionally stable product formula algorithms for nonlinear coupled thermoelasticity', Int. J. Plasticity, 9, 749-782 (1993). 
47. A. M. Cuitiño and M. Ortiz, 'A material-independent method for extending stress update algorithms from smallstrain plasticity to finite plasticity with multiplicative kinematics', Eng. Comput., 9, 437-451 (1992).

48. G. Weber and L. Anand, 'Finite deformation constitutive equations and a time integration procedure for isotropic hyperelastic-visoelastic solids', Comput. Methods Appl. Mech. Eng., 79, 173-202 (1990).

49. A. L. Eterovic and K. J. Bathe, A hyperelastic-based large strain elasto-plastic constitutive formulation with combined isotropic-kinematic hardening using the logarithmic stress and strain measures', Int. $j$. numer. methods eng., 39, 1099-1114 (1990).

50. R. J. Clifton and R. W. Klopp, 'Pressure-shear plate impact testing', Metals Handbook, 9th Edn, 8, $230-239$ (1985).

51. W. Tong, R. J. Clifton and S. Huang, 'Pressure-shear impact investigation of strain rate history effects in oxygen-free high-conductivity copper', J. Mech. Phys. Solids, 40, 1251-1294 (1992).

52. R.O. Ritchie and A. W. Thompson, 'On macroscopic and microscopic analyses for crack initiation and crack growth toughness in ductile alloys', Metall. Trans., 16A, 233-247 (1985).

53. R. O. Ritchie, J. F. Knott and J. R. Rice, 'On the relationship between critical tensile stress and fracture toughness in mild steel', J. Mech. Phys. Solids, 21, 395-410 (1973).

54. E. Orowan, 'Fracture and strength of solids', Rep. Prog. Phys., 12, 185-232 (1948).

55. J.F. Knott, 'Some effects of hydrostatic tension on the fracture behavior of mild steel', J. Iron Steel Inst., 204, 104-111 (1966).

56. F. Erdogan and G. C. Sih, 'On the crack extension in plates under plane loading and transverse shear', J. Basic Eng., 85, $519-527$ (1963).

57. H. C. Rogers, 'Tensile fracture of ductile metals', Trans. Metall. Soc. A.I.M.E., 218, 498-506 (1960).

58. J. Gurland and J. Plateau, 'Mechanism of ductile rupture of metals containing inclusions', Tran. Am. Soc. Metals, 56, 442-455 (1963).

59. T. B. Cox and J. R. Low, 'Investigation of the plastic fracture of AISI 4340 and 18 nickel-200 grade maraging steels', Metall. Trans., 5, 1457-1470 (1974).

60. J.Q. Clayton and J. F. Knott, 'Observations of fibrous fracture modes in a prestrained low-alloy steel', Metal. Sci., 10, 63-71 (1976).

61. Pellisier, G. E., 'Effect of microstructure on the fracture toughness of ultra-high strength steels', Eng. Fracture Mech., 1, 55-75 (1968).

62. G. Green and J. F. Knott, 'Initiation and propagation of ductile fracture in low steels', Trans. ASME 98, Series H, J. Engng Matl Tech., 37-46 (1976).

63. S. P. Rawal and J. Gurland, 'Observations on the effect of cementite particles on the fracture toughness of spheroidized carbon steels', Metall. Trans. A, 8A, 691-698 (1977).

64. J. R. Rice and M. A. Johnson, 'The role of large crack tip geometry changes in planes strain fracture', in $M$. F. Kannine, W. F. Adler, A. R. Rosendfield, and R. I. Jaffee, (eds.), Inelastic Behavior of Solids, 641 McGraw-Hill, New York, 1970.

65. J. R. Rice and D. M. Tracey, 'On the ductile enlargement of voids in triaxial stress fields', J. Mech. Phys. Solids, 17, $201-217(1969)$.

66. R. O. Ritchie, W. L. Server and R. A. Wullaert, "Critical fracture stress and fracture strain models for prediction of lower and upper shelf toughness in nuclear pressure vessel steels', Metall. Trans., 10A, 1557-1570 (1979).

67. M. Ortiz and J. J. Quigley, IV, 'Adaptive mesh refinement in strain localization problems', Comput. Methods Appl. Mech. Eng., 90, 781-804 (1991).

68. S. W. Sloan, 'A fast algorithm for constructing delaunay triangulations in the plane', Adv. Eng. Software, 9, 34-55 (1987).

69. J. C. Nagtegaal, D. M. Parks and J. R. Rice, 'On numerically accurate finite element solutions in the fully plastic range', Comput. Methods Appl. Mech. Eng., 4, 469-484 (1974).

70. A. M. Cuitiño, M. Ortiz, ‘Computational modelling of single crystals’, Modelling Simul. Mater. Sci. Eng., 1, 225-263 (1992).

71. X.P. Xu and A. Needleman, 'Numerical simulations of fast crack growth in brittle solids', J. Mech. Phys. Solids (to appear).

72. A. I. Shih and H. T. Y. Yang, 'Experimental and finite element predictions of residual stresses due to orthogonal metal cutring', Int. j. numer. methods eng., 36, 1487-1507 (1993). 